\title{
Predictors of the surgical outcome of propeller perforator flap reconstruction, focusing on the effective safe distance between the perforator and the wound edge
}

\section{Peng Wang \\ Wuxi No.9 People's Hospital Affiliated to Soochow University \\ Fang Lin \\ Wuxi No.9 People's Hospital Affiliated to Soochow University \\ YunHong Ma ( $\nabla 18205030286 @ 163 . c o m)$ \\ Wuxi No.9 People's Hospital Affiliated to Soochow University \\ Jianbing Wang \\ Wuxi No.9 People's Hospital Affiliated to Soochow University \\ Ming Zhou \\ Wuxi No.9 People's Hospital Affiliated to Soochow University \\ YongJun Rui \\ Wuxi No.9 People's Hospital Affiliated to Soochow University}

\section{Research Article}

Keywords: perforator flap, propeller flap, lower limb, risk factors

Posted Date: February 18th, 2021

DOI: https://doi.org/10.21203/rs.3.rs-200138/v1

License: (c) This work is licensed under a Creative Commons Attribution 4.0 International License.

Read Full License

Version of Record: A version of this preprint was published at BMC Musculoskeletal Disorders on July 29th, 2021. See the published version at https://doi.org/10.1186/s12891-021-04522-z. 


\section{Abstract}

Introduction: Soft tissue defects in the distal third of the leg and malleolus are difficult to cover and often require free tissue transfer, even for small-sized defects. Propeller flaps were designed as an alternative to free tissue transfer, but are reportedly associated with high complication rates. The aim of our study was to assess our institutional experience with the propeller flap technique and to predict its role in lower-limb reconstruction.

Methods: All patients who had undergone propeller flap reconstruction of a distal leg defect between 2013 and 2018 were included. Demographic, clinical, and follow-up data were analyzed.

Results: Complications occurred in 17 of 82 propeller flaps (20.7\%), comprising 11 cases of partial necrosis and six of total necrosis. There were no significant differences in age, sex, body mass index smoking, diabetes mellitus, and soft tissue defect sites between the groups of patients with versus without flap necrosis $(p>0.05)$. In the multivariable logistic regression

analysis with odds ratios (ORs) and $95 \%$ confidence intervals ( $95 \% \mathrm{Cls}$ ), there were also no significant differences between these two groups in the length and width of the fascial pedicle, and the ratio of the flap length to the flap width $(p>0.05)$. Interestingly, there were significant differences between the two groups in the distance between the flap perforator and the wound center $(p<0.05)$ and the rotation angle of the flap $(p<0.05)$. Receiver operating characteristic curve analysis showed that when the nearest distance from the flap to the wound was less than $3.5 \mathrm{~cm}$, the necrosis rate of the flap was markedly increased $(A \cup C=76.1)$; this suggests that the effective safe flap-wound distance was $3.5 \mathrm{~cm}$.

Conclusions: Propeller flaps are a reliable option for reconstruction in carefully selected patients with traumatic defects of the lower limb and malleolus. What we found is that What we found is that the 'effective safe distance' was $3.5 \mathrm{~cm}$ which the distance from the flap to the wound.

\section{Introduction}

The middle and lower limb and ankle contain a limited amount of soft tissue and have poor skin elasticity. Traumatic injuries to these regions often result in the exposure of bones and tendons, and it is difficult to directly close these defects, even for small defects. Therefore, flap covering is often required. In traumatic cases, the soft tissue coverage in this area is often further reduced due to extensive soft tissue destruction and the multiple incisions required for complex internal fixation. Moreover, the anatomical region of the internal fixation has limited soft tissue relaxation, which further increases the demand for soft tissue coverage. These factors often limit the use of traditional local flaps. With the development of flap surgery technology, Hyakusoku et al. [1] first proposed the concept of the "propeller flap" in 1991. The propeller flap is characterized by the subcutaneous fascia as the pedicle, with a flap at each end, giving a similar shape to a propeller; the flap rotates $90^{\circ}$ with the fascial pedicle as the axis to repair wounds on the elbow or axilla. In 2006, Hallock [2] proposed the fusion of the perforator flap and the propeller flap, and reported two cases (three flaps) in which the propeller flap pedicled with the adductor perforator was 
used to repair sacrococcygeal pressure sores. In 2009, the "Tokyo Consensus" of propeller flaps was formed at the first Tokyo Propeller Flap Conference [3]. The Tokyo Consensus defines a propeller flap as an island flap that repairs the recipient area through axial rotation. Any island flap can become a propeller flap; however, propelling island flaps and non-island rotating flaps are not considered propeller flaps.

The main advantages of the propeller flap for the treatment of calf defects include a short operation time, shortened hospital stay, reduced patient costs, and location of the flap sites adjacent to the defect sites $[4,5][6]$. The reported complication rates associated with propeller flaps range widely from 8.3 to $42 \%$ [614], and the main complications are venous congestion and partial flap necrosis. Partial necrosis of skin flaps is affected by patient factors (sex, age, defect location), flap factors (rotation point position, flap proximal position, flap length and width, fascial pedicle length and width, total flap length, aspect ratio of the flap), and operator factors. The necrosis rate of skin flaps is reportedly increased in elderly patients with diabetes mellitus and peripheral vascular disease $[15,16]$. In this study, we focused on the lower limb because partial necrosis are more frequent for PPF located on legs than in other locations. Furthermore, to reduce the flap necrosis rate, the flap perforator should not be too close to the wound surface or located too far from the wound. However, no study has quantitatively analyzed the closest safe distance between the perforator and the wound, that is, the effective safe distance.

We retrospectively analyzed the records of patients who underwent propeller flap treatment in our hospital to assess the distance from the perforator to the propeller flap, and determine the shortest safe distance from the perforator to the wound to increase the success rate and reduce the incidence of flap necrosis.

\section{Patients}

All adult patients who received propeller flap treatment from 2013 to 2018 were included in this singlecenter study. The study population comprised 82 patients ( 47 men and 35 women) with an average age of 36.5 years (range 18-65 years). All patients had soft tissue defects in the lower legs that required flap reconstruction. The indications for propeller flaps instead of free flaps included traumatic defects in the distal third of the leg, the need for flaps to cover an area less than $5 \mathrm{~cm}$ in diameter, perforator arteries that were detectable by Doppler, and unsuitability for complex microvascular surgery. The exclusion criteria were: age less than 18 years or more than 65 years; local peripheral vascular disease, chronic lymphedema, or deep vein thrombosis; other pathological defects; venous, neuropathic, and malignant ulcers; Gustilo type IIIC injuries. Patients with any of these exclusion criteria underwent free flap reconstruction rather than propeller flap reconstruction. Table 1 lists the demographic data of the patients. 
Table 1

Patient demographic data

\begin{tabular}{|ll|}
\hline Characteristics & Value \\
\hline No. of patients & 82 \\
\hline Mean age (range) & $36.5(18-65)$ \\
\hline Mean body mass index, kg/m & 22.5 \\
\hline Diabetes mellitus, no. (\%) & $12(14.6)$ \\
\hline Smoking, no. (\%) & $34(41.5)$ \\
\hline Previous radiotherapy, no. (\%) & $1(1.2)$ \\
\hline Defect location, no. & \\
\hline Medial malleolus & 31 \\
\hline External malleolus & 8 \\
\hline Lower tibia & 32 \\
\hline Middle tibia & 11 \\
\hline Cause of the defect, no. & \\
\hline Infection & 20 \\
\hline Trauma & 62 \\
\hline Gustilo-Anderson type (\%) & \\
\hline II & $6(7.3)$ \\
\hline IIIA & $19(23.2)$ \\
\hline IIIB & $57(69.5)$ \\
\hline Defect size (cm $\left.{ }^{2}\right)$ & 12.4 \\
\hline Source vessel & \\
\hline anterior tibial artery & \\
\hline posterior tibial artery & \\
\hline peroneal artery & \\
\hline Complications & \\
\hline Vascular crisis. no.(\%) & \\
\hline artery & \\
\hline
\end{tabular}




\begin{tabular}{|ll|}
\hline Characteristics & Value \\
\hline vein & 19 \\
\hline Infect. no.(\%) & $11(13.4)$ \\
\hline Superficial infection & 10 \\
\hline Deep infection & 1 \\
\hline Flaps necrosis. no. (\%) & $17(20.7)$ \\
\hline Partial necrosis & 11 \\
\hline total necrosis & 6 \\
\hline Aesthetic outcome & \\
\hline 5 & 2 \\
\hline 4 & 35 \\
\hline 3 & 37 \\
\hline 2 & 5 \\
\hline 1 & 3 \\
\hline
\end{tabular}

The criteria used to define survival of the skin flap were: the skin flap was fully alive, and the wound was healed at the first stage. Partial flap necrosis was defined as necrosis of less than $50 \%$ of the total flap area. The flap length was defined as the maximum length of the longitudinal axis of the flap; the flap width was defined as the maximum width of the flap; the total flap length was defined as the length of the flap plus the length of the fascia; the length-width ratio was defined as the ratio of the total flap length to the width of the fascial pedicle. Table 2 lists the factors indicative of flap success and failure. In receiver operating characteristic (ROC) curve analysis for the optimal cutoff value of the arc of rotation, maximal statistical significance was achieved at an arc of rotation threshold of $135^{\circ}$. Therefore, the patients were stratified into those with an arc of rotation of less than $150^{\circ}$ and those with an arc of rotation of $150-180^{\circ}$ for further comparative analysis [17]. The flap length was equal to the sum of the large oar and the small oar, and the length of the small oar was measured as the distance from the fulcrum to the wound surface. 
Table 2

Univariate analysis of the risk factors for vertebral compression fractures

\begin{tabular}{|c|c|c|c|}
\hline \multirow[t]{2}{*}{ Characteristics } & \multicolumn{2}{|c|}{ Flaps necrosis groups } & \multirow[t]{2}{*}{ p Value } \\
\hline & Yes & No & \\
\hline No. of flaps & 17 & 65 & \\
\hline Mean age, y & $45.9 \pm 9.3$ & $41.0 \pm 10.8$ & $0.094^{a}$ \\
\hline $\begin{array}{l}\text { Mean body mass } \\
\text { index, } \mathrm{kg} / \mathrm{m} 2\end{array}$ & $22.7 \pm 3.1$ & $22.3 \pm 3.0$ & $0.619^{a}$ \\
\hline Sex & & & $0.873^{b}$ \\
\hline Male & 8 & 32 & \\
\hline Female & 9 & 33 & \\
\hline Location & & & $0.676^{b}$ \\
\hline Middle tibia & 5 & 15 & \\
\hline Lower tibia & 7 & 26 & \\
\hline Lateral malleolus & 3 & 8 & \\
\hline Medial malleolus & 2 & 16 & \\
\hline Arc of rotation & & & $0.010^{b}$ \\
\hline$<150$ & 4 & 38 & \\
\hline $150-180$ & 13 & 27 & \\
\hline Diabetes mellitus & & & $0.693^{b}$ \\
\hline Yes & 3 & 9 & \\
\hline No & 14 & 56 & \\
\hline Smoking & & & $0.562^{b}$ \\
\hline Yes & 6 & 28 & \\
\hline No & 11 & 37 & \\
\hline Distance from perforator to center of defect & $8.4 \pm 2.3$ & $9.4 \pm 2.6$ & $0.151^{c}$ \\
\hline Nearest distance from perforator to defect location & $3.0 \pm 0.9$ & $3.8 \pm 1.0$ & $0.005^{\mathrm{c}}$ \\
\hline
\end{tabular}




\begin{tabular}{|lccc|}
\hline Characteristics & \multicolumn{2}{c}{ Flaps necrosis groups } & p Value \\
\hline $\begin{array}{l}\text { Time from injury to definitive surgical } \\
\text { procedure (days) }\end{array}$ & $11.2 \pm 4.2$ & $10.0 \pm 3.5$ & $0.220^{c}$ \\
\hline Operative time (min) & $160.3 \pm 23.7$ & $166.5 \pm 18.7$ & $0.256^{c}$ \\
\hline Length(cm) & $12.4 \pm 3.2$ & $11.8 \pm 3.7$ & $0.573^{c}$ \\
\hline Width $(\mathrm{cm})$ & $5.6 \pm 2.1$ & $6.2 \pm 1.4$ & $0.428^{c}$ \\
\hline Flap Size $(\mathrm{cm})$ & $62.5 \pm 14.6$ & $58.9 \pm 13.5$ & $0.317^{c}$ \\
\hline a independent t-test; ${ }^{\mathrm{b}}{ }^{\mathrm{C}}$ Chi-square tests; ${ }^{\mathrm{C}}$ Mann-Whitney U tests * $P<0.05$. & \\
\hline
\end{tabular}

\section{Surgical steps}

\section{Preoperative positioning}

Preoperative planning was done using CTA and/or color Doppler ultrasound to locate the perforating vessels.

\section{Skin flap design}

(1) First, the large propeller of the proximally designed propeller flap was cut and rotated to cover the soft tissue defect of the recipient area; the skin from the distal end of the perforator to the wound surface of the recipient area was designed as a spiral. The small paddle covered part of the donor site wound after rotation (Fig. 1). (2) The size of the propeller flap consisted of the size of the wound and the position of the perforator. The length of the flap was equal to the sum of the large oar and the small oar. The length of the large oar was slightly larger than the sum of the length of the long axis of the wound and the length of the small oar. The flap width was slightly larger than the wound width; the length and aspect ratio of the large oar and the small oar were the same as for the originally described pedicled perforator flap. The pedicle perforator flap was used as a reference and did not exceed the cutting range. (3) The axis of the flap conformed to the previous island flap or neurocutaneous nutrition. (4) The shape of the flap was reversed at the end of the oar in accordance with the shape of the wound. (Figs. 2)

\section{Intraoperative steps}

Based on the preoperative identification of the perforator positions and the flap design, the skin was incised along one side of the flap, and separated on the surface or deep surface of the fascia. All perforators were initially saved, and the best perforator was then chosen as the vascular pedicle. The length and position of the flap were adjusted in accordance with the actual position of the perforator. The other side of the flap was cut and the perforator was separated. After thorough hemostasis, the flap was 
rotated, and the vascular pedicle was checked to ensure that there was no compression, twisting, stretching, or bleeding. The flap was sutured, drainage was placed, and local bracing was performed.

\section{Postoperative treatment}

After the operation, appropriate antibiotics were selected based on the bacterial culture and drug sensitivity results. If the bacterial culture was negative, empirical broad-spectrum antibiotics were administered. An intramuscular injection of papaverine $(30 \mathrm{mg})$ was routinely administered every 8 hours to prevent vasospasm; patients also received adequate fluid replacement, and monitoring of the central vein pressure. Prophylactic anticoagulation therapy was performed for 3-5 days postoperatively. The swelling, color, elasticity, and capillary reaction of the skin flap were closely observed.

\section{Complication management}

If there was venous stasis (shown as swelling of the skin flap, purple color, and an accelerated capillary reaction), we first determined whether the skin sutures of the vascular pedicle were too tight and there was too much local tension. If necessary, some or all of the vascular pedicle sutures were removed. If suture removal did not improve the venous stasis, blood dripping therapy was implemented immediately; two to three 5-mm incisions were made on the edge of the skin flap, and heparin solution $(25 \mathrm{U} / \mathrm{ml})$ diluted with normal saline was administered to maintain incisional bleeding. Patients were closely observed to ensure that blood dripping therapy did not cause excessive blood loss. If skin flap necrosis occurred, the wound was debrided to remove the necrotic tissue, and the activity of the deep fascia of the skin flap was assessed. If the deep fascia was viable, vacuum sealing drainage dressing was applied to the wound, and free skin grafting was performed in second-stage surgery; if the fascia was necrotic, the fascia was removed, vacuum sealing drainage dressing was applied, and the wound was covered with a flap in second-stage surgery. If necrosis occurred after the purse-pack was removed by skin grafting in the donor area, or the wound of the donor area was directly sutured and the cut edge was necrotic, the necrotic tissue was removed and the deep soft tissue bed was scraped with a curette until the appearance of freshly oozing blood. External dressings were applied and changed until the wound healed.

\section{Statistical analysis}

Statistical analyses were performed using SPSS 23.0 software. Measurement data are expressed as mean \pm standard deviation, and compared using the Student's t-test or the Mann-Whitney U test; count data were compared using the $\chi^{2}$ test or Fisher's exact test. Multivariate logistic regression was used to analyze the factors influencing the occurrence of partial flap necrosis. $P<0.05$ was considered statistically significant.

\section{Results}

Clinical results 
All 82 patients were followed up after surgery. The mean follow-up time was $12.5 \pm 4.2$ months (range 3 to 36 months). The flaps completely survived and the wounds healed well in 65 of 82 patients (79.3\%); there were 11 cases of partial necrosis (13.4\%), and six of complete necrosis (7.3\%). Venous crisis occurred in 19 of 82 patients (23.2\%); active treatment rescued the flaps in four patients, while the flaps developed necrosis in 15 patients. Arterial crisis occurred in two cases, comprising one case of flap necrosis, and one case in which the flaps were successfully rescued. Deep infection developed in one patient $(1.2 \%)$ who subsequently developed complete skin flap necrosis. Superficial infection developed in 10 patients $(12.3 \%)$. Using the posterior tibial artery perforator fascia pedicle flap alone or in combination with simple measures, including dressing changes, secondary suturing, and skin grafting, $91.53 \%$ of the wounds were repaired. The donor site healed in the first stage postoperatively, and there were no complications such as ulcers, skin graft necrosis, bone scars, and joint contractures. The results are shown in Table 1, and typical cases are shown in Figs. 2 .

Comparison of the flap survival group and necrosis group (including partial and total necrosis)

There were 65 patients in the flap survival group and 17 in the necrosis group. The comparison of the two groups is shown in Table 2. There were no significant differences between the two groups in age, sex, body mass index smoking, prevalence of diabetes, and soft tissue defects $(P>0.05)$. There were no significant differences between the two groups in the length and width of the fascial pedicle, length and width of the flap, and aspect ratio of the flap $(P>0.05)$. The distance between the flap perforator and the wound center did not significantly differ between groups. However, the two groups significantly differed regarding the shortest distance between the skin flap perforator and the wound edge $(P<0.05)$, and the rotation angle of the flap $(P<0.05)$.

Multivariate analysis

Table 3 shows the results of the multivariate analysis using the dichotomous variable of whether partial necrosis of the skin flap occurred as the dependent variable, and the other variables as independent variables. The shortest distance between the flap perforator and the wound edge was a risk factor for partial necrosis of the flap $(P<0.05)$.

Table 3

Multiple logistic regression analysis of the risk factors for propeller flap necrosis

\begin{tabular}{|llll|}
\hline Variables & Odds-ratio & $95 \% \mathrm{Cl}$ & P-value \\
\hline Nearest distance from perforator to defect location & 0.806 & $0.854-0.952$ & 0.000 \\
Arc of rotation & 2.829 & $1.284-6.670$ & 0.016 \\
\hline
\end{tabular}

ROC curve analysis

ROC curve analysis showed that when the closest distance between the perforating branch of the skin flap and the wound surface was less than $3.5 \mathrm{~cm}$, the necrosis rate of the skin flap was significantly 
increased. The results are shown in Table 4 and Fig. 3.

Table 4

Sensitivity, specificity, AUC, and cutoff values of the predictors for propeller flap necrosis

\begin{tabular}{|lccccc|}
\hline Variable & Sensitivity & Specificity & AUC* & Cutoff & $\begin{array}{l}\text { P- } \\
\text { value }\end{array}$ \\
\hline $\begin{array}{l}\text { Nearest distance from perforator to defect } \\
\text { location }\end{array}$ & $69.7 \%$ & $82.4 \%$ & $76.1 \%$ & 3.50 & 0.005 \\
\hline *Area under the curve. & & & & & \\
\hline
\end{tabular}

\section{Discussion}

A perforator flap is a type of skin flap or subcutaneous flap that is supplied by one (or more) perforator blood vessels that branch from a deeper blood vessel. The isolated perforator is moved and freely dissected together with the overlying tissue, enabling the flap to be moved. Simple translation or transposition of the flap is sufficient in some cases, but when the flap needs to be rotated by more than $90^{\circ}$, it is usually deployed in the manner of a propeller with the perforator used as the axis of rotation, which is called a propeller flap [3].

The propeller flap is a special form of the perforator flap. The advantages of the propeller flap are as follows: (1) because the donor site is located near the defect, the flap is composed of tissue that is similar to the tissue of the recipient site; (2) the donor site arteries and muscles are used to completely or partially close the donor site defect and reduce the morbidity rate of the donor site; (3) lower technical requirements and faster transfer than free tissue flap reconstruction [18-22]. The propeller flap is gaining popularity as a reliable technique for repairing soft tissue defects in the distal leg and ankle; the postoperative appearance is satisfactory, and the procedure is simple, easy to master, and requires a short operation time [11,24]. The main source arteries for the propeller flap in the distal leg are the posterior tibial artery, anterior tibial artery, and peroneal artery [11].

Although the propeller flap is an established surgical technique, serious complications may still occur and cause failure if not adequately addressed. As the propeller flap is a type of perforator flap, the occurrence of venous return disorder cannot be completely avoided. Venous return disorder is the most common complication of propeller flaps, and is one of the main causes of flap necrosis. Necrosis mainly occurs at the distal end of the flap, but may lead to necrosis of the entire flap in severe cases. Flap necrosis reportedly occurs in $10.77-24.00 \%$ of cases [25-28]. In the present study, the rate of partial flap necrosis was $20.7 \%$. Flap necrosis may be more likely to occur when the flap perforator is located in the injured area, but this hypothesis lacks objective evidence. There are many reasons for skin flap necrosis. It is currently believed that propeller flap necrosis is influenced by the flap size, pedicle length, and angle of rotation $[3,29]$. 
The first factor causing propeller flap necrosis is the flap size. The size of the propeller flap, especially the size of the large paddle, has a large effect on venous return. After the flap is rotated, if the length of the large paddle is insufficient, the flap is stretched so that it barely covers the wound and causes excessive tension of the vascular pedicle. In addition, flap rotation and other factors increase the risk of venous return disorder. Adequate preoperative preparation and flap design effectively reduce this problem. In the present study, preoperative Doppler examination was performed to determine the locations of the perforating vessels in the distal leg. The rotation point of the perforator propeller flap depends on the position of the perforator fulcrum. In theory, the closer the perforator fulcrum is to the wound, the greater the length of the flap that can be cut. The size of the skin flap should be designed in accordance with the size of the wound, and the position and diameter of the perforating vessels. The large paddle is located near the axis of rotation, and its length should be 0.5 to $1.0 \mathrm{~cm}$ longer than the distance from the point of rotation to the most distal end of the wound. The small paddle is located between the rotation point and the wound surface. The width of the flap should be $0.5-1.0 \mathrm{~cm}$ wider than the wound surface, and is determined by the thickness of the subcutaneous fat. However, the closer the perforator is to the wound, the greater the impact on the wound. Wound inflammation reportedly damages the perforator blood vessels and causes necrosis [30]. Our study found that the necrosis rate was significantly higher when the perforator was farther away from the flap. Furthermore, ROC curve analysis showed that the flap necrosis rate increased when the distance from the perforator to the wound was less than $3.5 \mathrm{~cm}$ (sensitivity 69.7\%; specificity 82.4\%), Table 4). We consider that there were three reasons for the increased risk of flap necrosis in cases where the perforator was closer to the flap. 1. The degree of inflammation. The inflammatory response of the flap is related to the injury mechanism/wound contamination severity. In the present study, the injuries were caused by high-energy trauma, while contaminated skin defects were excluded. 2. The shape of the skin wound. The present study assessed the distance from the perforator to the center of the flap, while the distance from the perforator to the wound center did not significantly affect the flap necrosis rate (Fig. 2B). 3. The time from injury to wound coverage, and the inflammatory reaction period. As inflammation peaks at 7-12 days after injury, it is optimal to cover the wound before or after this period. The exposed wound needs to be treated with standard dressing changes.

Previous studies have also shown that the flap width affects the survival of the flap, as the anastomoses between the perforators of the main blood vessels in the calf are almost all choke anastomoses [31]. Therefore, the wider the flap, the farther the edge of the flap will be from the axis of rotation, and the greater the decrease in the diameter of the vascular network and the pressure of the blood flow; furthermore, due to the special anatomical structure of the lower leg, when the flap position is lower, the wider edge of the flap approaches or even surpasses the midline of the front and rear of the calf, which directly leads to partial necrosis of the flap.

The second factor affecting propeller flap necrosis is the length of the vascular pedicle. After the flap is rotated, the pedicle must be kept under appropriate tension. A vascular pedicle that is too short causes excessive local tension due to traction, which causes venous return disorder. Intraoperatively, the pedicle vessel should be dissected in the direction of the source vessel as much as possible to create a length of 
at least $3 \mathrm{~cm}$ and a width of at least $1 \mathrm{~mm}$ [32]; this significantly reduces the risk of blood vessel deformation after rotation. The caliber of the blood vessel must also be considered. Preoperative Doppler examination must be performed to locate the perforator position and select a perforator with a suitable caliber as the direct nutrient vessel for the flap [33].

The third factor affecting propeller flap necrosis is the flap rotation angle. The propeller flap needs to rotate at a large angle of up to $180^{\circ}$. The perforating vessels, especially the perforating veins, are easily compressed by the surrounding deep fascia fiber bundles due to their thin wall and low pressure.

Therefore, the vascular pedicle usually needs to be naked, and different rotation directions (clockwise or counterclockwise) should be assessed intraoperatively; the rotation direction that causes the smallest twist of the pedicle should be selected [35]. The complication rate of propeller flap reconstruction is higher in the extremities than in the trunk. This is because the trunk has relatively abundant perforators and large perforator areas connected by blood vessels, which may aid in the safe harvest of flaps, thereby reducing the incidence of complications [36-41].

Finally, previous studies suggest that other risk factors for flap necrosis include patient age over 50 years, smoking history, and diabetes mellitus [6,29], but these factors did not significantly affect the development of flap necrosis in the present study.

This study has some limitations. First, the sample size was relatively small, so a significant relationship between potential risk factors and complications may be hidden. Second, this was not a prospective study. Third, we did not perform this kind of surgery on a series of patients with bone defects in other parts, which means that there may be selective bias in choosing this surgical technique. Fourth, there was no control group who underwent other surgical techniques, such as free flap reconstruction. However, although we did not compare the results of perforator and free flap reconstructions, a recent metaanalysis study reported that the overall failure and complication rates are similar for free flaps $(19.0 \%)$ and perforator flaps (21.4\%) [42]. Despite these study limitations, to the best of our knowledge, this is the first quantitative and accurate study of the relationship between the position of the propeller flap and the wound surface. This research will help surgeons identify potential risk factors and choose the appropriate surgical method to repair soft tissue defects of the lower limbs.

\section{Conclusion}

The propeller flap has been widely used in reconstruction of defects on the trunk and limbs. The present study showed that when the distance between the flap perforator and the wound surface is less than 3.5 $\mathrm{cm}$, the necrosis rate of the flap is significantly increased; that is, the effective safe distance is $3.5 \mathrm{~mm}$. When the perforator is within this distance, the propeller skin needs to be selected more carefully.

\section{Limitations}


Firstly, this was a retrospective study, and small sample size. Indeed it is possible that an association between risk factors and complications might have been missed due to insufficient sample size. Nevertheless, our data may be considered as useful pilot data for fu-ture, multicenter studies on this topic.

\section{Declarations}

\section{Acknowledgements}

We thank the support from the Editage in language editing.

\section{Funding}

No funds were received in support of this study.

\section{Contributions}

Peng Wang design and drafting of manuscript. Fang Lin wrote the main manuscript text. Peng Wang and Fang Lin have contributed equally to this work

Jianbing Wang, MD, Ming Zhou, Data collection (radiographic parameters).

Corresponding author: Yunhong Ma and Yongjun Rui have contributed equally to this work, Surgical operation and statistical analysis. Yunhong Ma revision of manuscript.

All authors reviewed the manuscript.

\section{Ethics declarations}

All procedures performed in studies involving human participants were in accordance with the ethical standards of the institutional and/or national research committee and with the 1964 Helsinki declaration and its later amendments or comparable ethical standards.

This study was approved by Wuxi No.9 People's Hospital Affiliated to Soochow University ethics committee. We obtained written informed consent from all the participants before publishing this information

\section{Consent for publication}

Not applicable.

\section{Competing interests}

Peng Wang, Fang Lin, Yunhong Ma, Jianbing Wang, Ming Zhou, Yongjun Rui declare that they have no conflict of interest. 
Availability of data and materials

The datasets used and/or analyzed during the current study available from the corresponding author on reasonable request.

\section{References}

1. Hyakusoku H, Yamamoto T, Fumiiri M. The propeller flap method. Br J Plast Surg 1991;44(1):53-54.

2. Hallock GG. The proprller flap version of the adductor muscle perforator flap for coverage of ischial or trochanteric pressure sores. Ann plast surg, 2003, 56(5): 540-542.

3. Pignatti M, Ogawa R, Hallock GG, et al. The "Tokyo" consensus on propeller flaps. Plast Reconstr Surg. $2011 \mathrm{Feb} ; 127(2): 716-22$.

4. Sisti A,D'aniello C,Fortezza L,et a1.Propeller flaps: a literature review.In Vivo,2016,30(4):351-373.

5. Bekara F,Herlin C,Somda S,et a1.Free versus perforator-pedicled propeller flaps in lower extremity reconstruction: What is the safest coverage? A meta-analysis.Mierosurgery. 2018; 38(1):109-119.

6. Tos $\mathrm{P}$, Innocenti $\mathrm{M}$, Artiaco $\mathrm{S}$, et al. Perforator-based propeller flaps treating loss of substance in the lower limb. J Orthop Traumatol. 2011 Jun;12(2):93-9.

7. ScaglioniMF, Giuseppe AD, Chang El. Propeller flap reconstruction of abdominal defects: review of the literature and case report. Microsurgery 2015;35(1):72-78.

8. D'Arpa S, Cordova A, Pignatti M,et al. Freestyle pedicled perforator flaps: safety, prevention of complications, and management based on 85 consecutive cases. Plast Reconstr Surg 2011; 128(4):892-906.

9. Ioannidis S, Spyropoulou GA, Sadigh P, et al. Pedicledfree-style perforator flaps for trunk reconstruction: a reliable method. Plast Reconstr Surg 2015;135(2):602-609.

10. Innocenti M, Menichini G, Baldrighi $C$, et al. Are there risk factors for complications of perforatorbased propeller flaps for lower-extremity reconstruction? Clin Orthop Relat Res 2014;472(7):22762286

11. Gir P, Cheng A, Oni G, et al. Pedicled-perforator (propeller) flaps in lower extremity defects: a systematic review. J Reconstr Microsurg 2012;28(9):595-601

12. Nelson JA, Fischer JP, Brazio PS, et al. A review of propeller flaps for distal lower extremity soft tissue reconstruction: Is flap loss too high? Microsurgery 2013;33(7): 578-586

13. Oh TS, Hallock G, Hong JP. Freestyle propeller flaps to reconstruct defects of the posterior trunk: a simple approach to a difficult problem. Ann Plast Surg 2012;68(1):79-82

14. Lazzeri D, Huemer GM, Nicoli F, et al. Indications, outcomes, and complications of pedicled propeller perforator flaps for upper body defects: a systematic review. Arch Plast Surg 2013;40(1): 44-50.

15. Parrett B, Pribaz JJ, Matres E, et al. Risk analysis for the reverse sural fasciocutaneous flap in distal leg reconstruction [J]. Plast Reconstr Surg, 2009, 123 (5) : 1499-1504. 
16. Baumeister SP, Spierer R, Erdmann D, et al. A realistic complication analysis of 70 sural artery flaps in a multimorbid patient group[J]. Plast Reconstr Surg, 2003, 112 (1) : 129-140.

17. Joo Myong Paik, Jai-Kyong Pyon. Risk Factor Analysis of Freestyle Propeller Flaps. J Reconstr Microsurg 2017;33:26-31.

18. Koshima I, Moriguchi T, Ohta S, at al. The vasculature and clinical application of the posterior tibial perforator-based flap. Plast Reconstr Surg. 1992 90:643-649.

19. Jakubietz RG, Jakubietz MG, Gruenert JG,et al. The 180-degree perforatorbased propeller flap for soft tissue coverage of the distal, lower extremity: a new method to achieve reliable coverage of the distal lower extremity with a local, fasciocutaneous perforator flap. Ann Plast Surg. 2007; 59:667-671, 2007.

20. Jakubietz RG, Jakubietz DF, Gruenert JG, et al. Reconstruction of soft tissue defects of the Achilles tendon with rotation flaps, pedicled propeller flaps and free perforator flaps. Microsurgery.2010; 30:608-613.

21. Pignatti $M$, Pasqualini $M$, Governa $M$,et al. Propeller flaps for leg reconstruction. J Plast Reconstr Aesthet Surg.2008; 61:777-783.

22. Hallock GG. A paradigm shift in flap selection protocols for zones of the lower extremity using perforator flaps. J Reconstr Microsurg 2013; 29:233-240.

23. Gir P, Cheng A, Oni G, et al. Pedicled-perforator (propeller) flaps in lower extremity defects: a systematic review. J Reconstr Microsurg 2012;28(9):595-601.

24. Georgescu AV. Propeller perforator flaps in distal lower leg: evolution and clinical applications. Arch Plast Surg 2012;39(2):94-105

25. Nenad T, Reiner W, Michael S, et al. Saphenous perforator flap for reconstructive surgery in the lower leg and the foot: a clinical study of 50 patients with posttraumatic osteomyelitis. J Trauma 2010; 68 (5): $1200-1207$.

26. Dai JZ, Chai YM, Wang CY, et al. Distally based saphenous neurocutaneous perforator flap for reconstructive surgery in the lower leg and the foot: a long-term follow-up study of 70 patients [ $\mathrm{J}] . \mathrm{J}$ Reconstr Microsurg 2013; 29 (7): 481-486.

27. M.A. Hifny, A.M.A. Tohamy;O. Rabie, et al. Propeller perforator flaps for coverage of soft tissue defects in the middle and distal lower extremities. Ann Chir Plast Esthet $2020 ; 65(1): 54-60$.

28. Gunnarsson GL, Jackson IT, Westvik TS, et al. The freestyle pedicle perforator flap: a new favorite for the reconstruction of moderate-sized defects of the torso and extremities. Eur J Plast Surg 2015;38:31-36.

29. Bekara F, Herlin C, Mojallal A, et al. A Systematic Review and Meta-Analysis of Perforator-Pedicled Propeller Flaps in Lower Extremity Defects: Identification of Risk Factors for Complications. Plast Reconstr Surg 2016;137(1):314-331

30. Nikhil Panse, Parag Sahasrabudhe. Free style perforator based propeller flaps: Simple solutions for upper extremity reconstruction!. Indian J Plast Surg. 2014; 47(1); 77-84. 
31. Gascoigne AC, Taylor GI, Corlett RJ, et al. The relationship of superficial cutaneous nerves and interperforator connections in the leg: a cadaveric anatomical study. Plast Reconstr Surg 2017; 139 (4): 994e-1002e.

32. Wong $\mathrm{CH}$, Cui F, Tan BK, et al. Nonlinear finite element simulations to elucidate the determinants of perforator patency in propeller flaps. Ann Plast Surg 2007;59(6):672-678.

33. Jason K.F. Wong, Nidal Deek, Chung-Chen Hsu, et al. Versatility and "flap efficiency" of pedicled perforator flaps in lower extremity Reconstruction. Journal of Plastic, Reconstructive \& Aesthetic Surgery (2017) 70, 67-77.

34. Beniamino Brunetti, Stefania Tenna, Igor Poccia, et al. Propeller Flaps With Reduced Rotational Angles: Clinical Experience on 40 Consecutive Reconstructions Performed at Different Anatomical Sites.Ann Plast Surg. 2017;78(2):202-207.

35. Rout DK, Nayak BB, Choudhury AK, et at. Reconstruction of high voltage electricburn wound with exposed shoulder jioint by thoracoacromial artery perforator propeller flap. Indian J Plast Surg 2014, 47(2): 256-258.

36. Saint-Cyr M, Wong C, Schaverien M, et al. The perforasome theory: vascular anatomy and clinical implications. Plast Reconstr Surg 2009;124(5):1529-1544.

37. Schmidt M, Tinhofer I, Duscher D, et al. Perforasomes of the upper abdomen: an anatomical study. J Plast Reconstr Aesthet Surg 2014;67(1):42-47.

38. Ioannidis S, Spyropoulou GA, Sadigh P, et al. Pedicled free-style perforator flaps for trunk reconstruction: a reliable method. Plast Reconstr Surg 2015;135(2):602-609ã⿰口

39. Lazzeri D, Huemer GM, Nicoli F, et al. Indications, outcomes, and complications of pedicled propeller perforator flaps for upper body defects: a systematic review. Arch Plast Surg 2013;40(1):44-50.

40. Gunnarsson GL, Jackson IT, Westvik TS, et al. The freestyle pedicle perforator flap: a new favorite for the reconstruction of moderate-sized defects of the torso and extremities. Eur J Plast Surg 2015;38:31-36.

41. Park SW, Oh TS, Eom JS,et al. Freestyle multiple propeller flap reconstruction (jigsaw puzzle approach) for complicated back defects. J Reconstr Microsurg 2015;31(4): 261-267.

42. Bekara F, Herlin C, Somda S, et al. Free versus perforator-pedicled propeller flaps in lower ex-tremity reconstruction: what is the safest coverage? A meta-analysis. Microsurgery 2018;38(1):109-19.

\section{Figures}




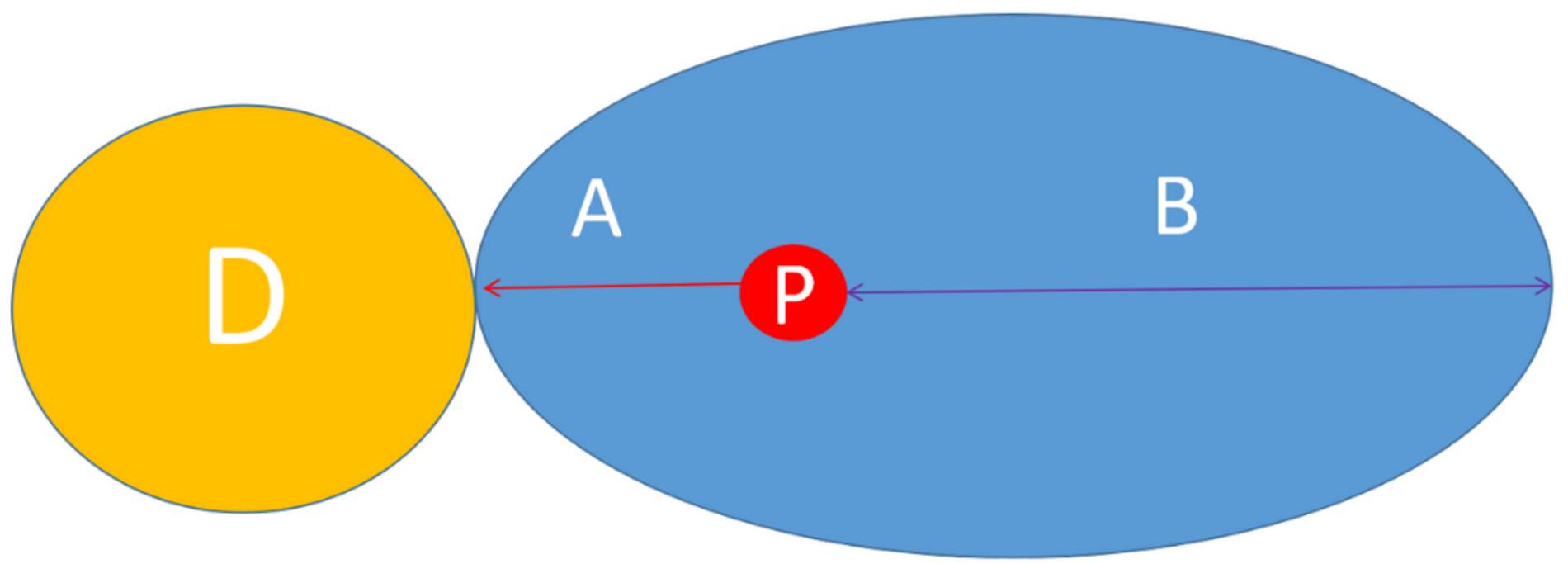

Figure 1

Drawing depicting a propeller flap. The distance of A (Nearest distance from the perforator to the defect) in a propeller flap. A flap rotation of 180 degrees is possible if there is available tissue in the linear axis (A) connecting the defect (D) (yellow area) and the emerging point of the perforator vessel $(P)$ (red circle), on the other side of the perforator, compared with the position of the $\operatorname{defect}(\mathrm{B})$ (blue area). 


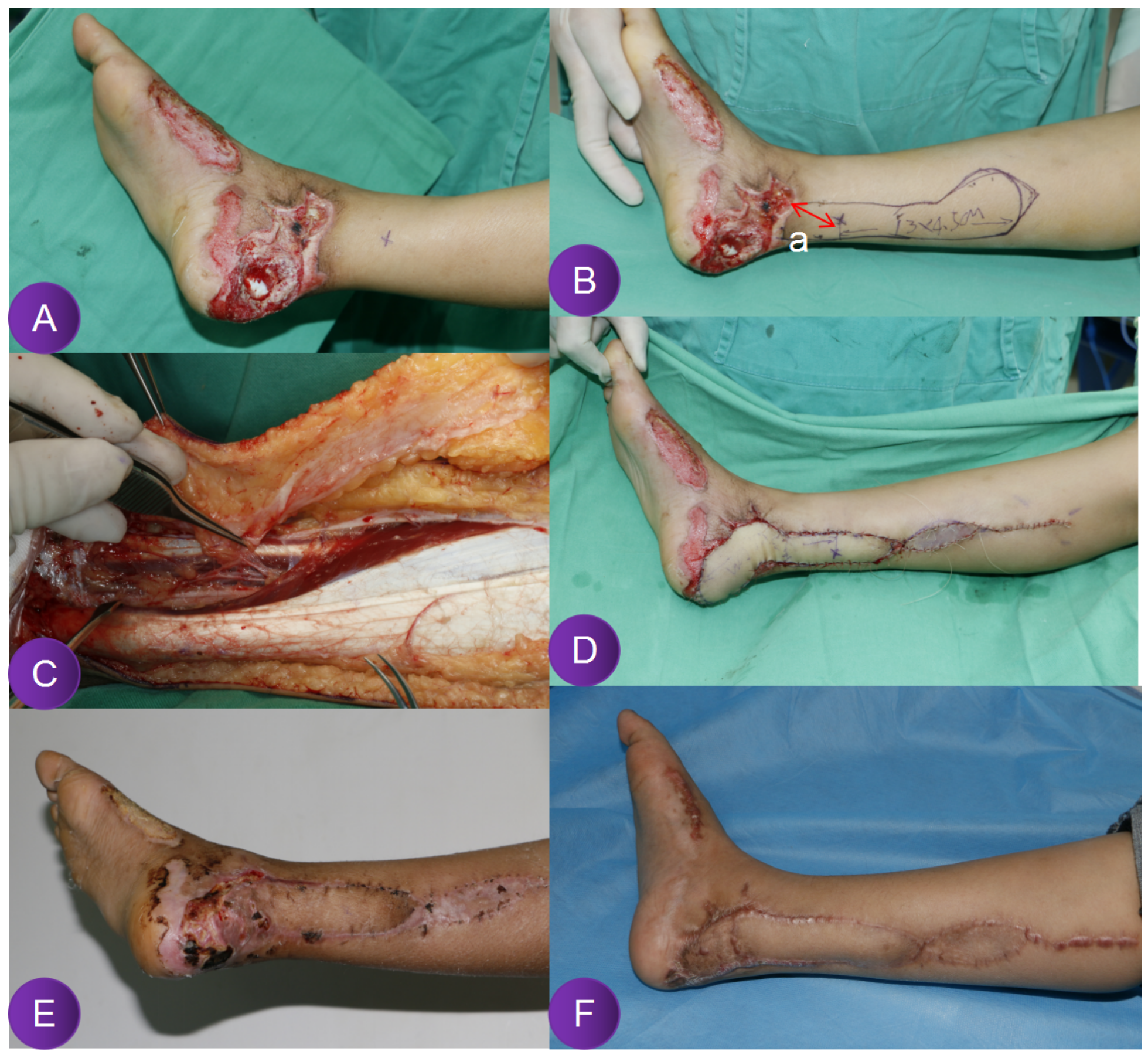

Figure 2

Photographs illustrating the propeller flap operative technique. A, B) Case example showing typical locations and marking of perforators from the posterior tibial arteries. C) The incision along one margin of the proposed flap. D) Final defect coverage with the propeller flap and primary closure of the donor site. E) Partial flap necrosis at 3 weeks postoperatively. F) Healing and excellent contour of the reconstructed ankle at 6 months postoperatively. a: Nearest distance from the perforator to the defect. 


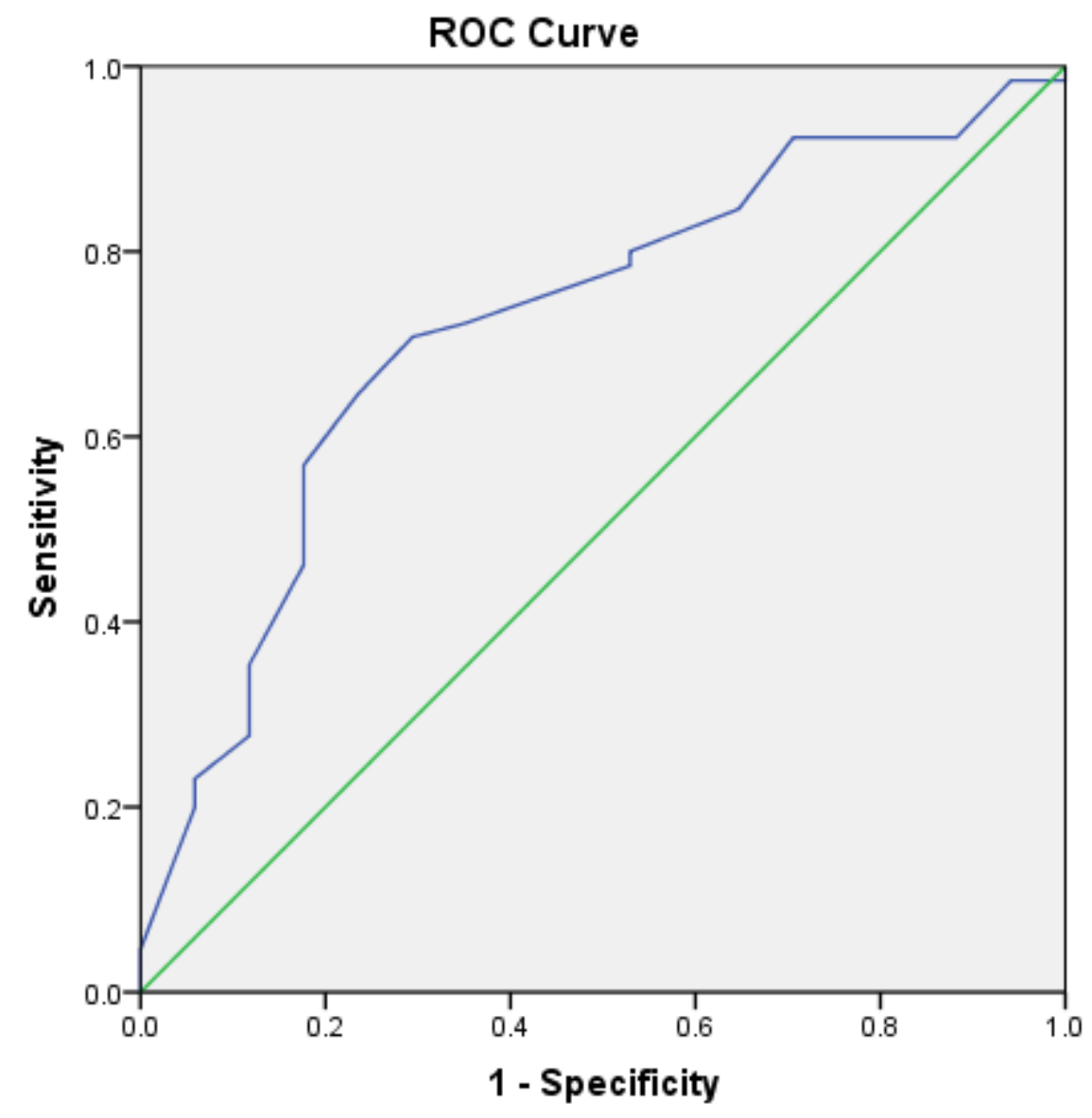

Diagonal segments are produced by ties.

\section{Figure 3}

Receiver operating characteristic curves. The optimal cutoff value of nearest distance from the perforator to the defect is shown for prediction of surgical outcome. 